\title{
A Different Approach for Big Bang Singularity
}

\section{Malay Kanti Sikdar}

Department of Physics, University of Kalyani, Kalyani, Nadia, W. Bengal, India

Correspondence to: M. K. Sikdar, sikdarmalay@ rediffmail.com

Keywords: Big Bang, Singularity, Gram, Manifestation of the Universe, Dissolution \& Scripture

Received: December 30, $2017 \quad$ Accepted: April 8, $2018 \quad$ Published: April 11, 2018

Copyright $\odot 2018$ by author and Scientific Research Publishing Inc.

This work is licensed under the Creative Commons Attribution-NonCommercial International License (CC BY-NC 4.0). http://creativecommons.org/licenses/by-nc/4.0/

\section{(c) (i) (9) Open Access}

\section{ABSTRACT}

With the discovery of Hubble Red Shift in the cosmological Universe, the question of Big Bang explosion has become inevitable and to explain this point of singularity thereon. Dissolution and manifestation of the universe happen to occur periodically. In this paper, analysis has been undertaken on the basis of scientific development on well accepted cosmological model, different scientific concepts and on one Vedic/Tantric scriptural remark. According to Vedic/Tantric scriptural remark, at the time of dissolution of the universe it remains in the form of gram. Different scientific tools like Electron Microscopy, X-rays powder diffraction method. Raman \& UV spectroscopy have been applied in analyzing the two parts of gram and finally to find out what do they correspond to cosmological analysis of Big Bang singularity. Main mechanisms in action in dissolution and manifestation have also been pointed out.

\section{INTRODUCTION}

With the discovery of Hubble Red Shift, one starting point of the universe has become unavoidable which is singular in nature i.e. indescribable \& indefinable. Normally it is thought that before Big Bang explosion the entire universe remains concealed in a smallest of the small points and the meaning of space and time was suspended. Evolution of the cosmological universe was started from the point of singularity by a big explosion [1-5]. Our universe is considered to have begun from an infinitesimally small, infinitely hot, infinitely dense point which is something known as big bang singularity where the all physical laws are broken down, even the Einstein theory of relativity does not work. At the time of Big Bang, all the materials and the radiation are crashed into a point of infinite density wherein physical \& chemical laws as we know become ineffective and even then we don't know yet what that point really is! Questions are many. Did the universe begin with singularity? Is it a point in space or a moment in time where everything in the universe was crushed together into infinitely hot or dense pocket known as singularity? For that reason, Big Bang is known as initial singularity. Many theories have been developed to explain the origin of the universe [6]. After Big Bang explosion from an ignition point material flies and the universe starts appearing by the process of evolution. Unfortunately, for Big Bang things aren't so simple, and in a sense the 
answer is everywhere and nowhere. The first few minutes of big bang explosion are followed by plasma phase lasting of the order of $10^{5}$ years during which the universe was radiation dominated and the baryonic gas consisting almost likely of hydrogen, helium, was ionized and consequently become opaquest to radiation through electron scattering. But the expansion was accompanied by cooling and when the temperature has come down to $104 \mathrm{~K}$ the matter began to dominate about $37 \times 10^{4}$ years after Big Bang, first hydrogen become neutral helium by combination, rest by nucleosynthesis [7] and capture of electrons. The back ground radiation became scattered for last time at $2.7 \mathrm{~K}$. Eventually gas began to settle in certain points resulting formation of stars, galaxies etc. It is needless to mention that life is revealed at molecular level of evolution [8]. Of course within Plank Time $5.31 \times 10^{-4} \mathrm{~s}$ the situation was not so simple. With formation of huge sub-atomic quasi particles (Baryon, quark etc) etc. as intermediate phase huge neutrino were also formed. Edwin Hubble (1889-1953) on Mount Wilson where he did much of research that led him to propose that the universe is expanding. Hubble was able to show that there are many galaxies just like our milky way, composed of hundred billions of stars; which are moving away and observed a red shift in spectral analysis of the light coming out of them. He deduced a law, if $d$ is the distance of the galaxy from the earth (or any other point of reference in the universe) and $\mathrm{V}$ is the velocity with which the galaxy appears to be moving away from us, Hubble Law gives:

$$
V=H d
$$

where $H$ is a proportionality constant known as Hubble parameter. The Hubble parameter has dimension of inverse time. We must independently deduce the distance of the galaxy from the earth. The recessional speed can be measured straight way by using Doppler shift of light from the galaxy, $H=72 / \mathrm{Km} / \mathrm{sec} / \mathrm{Mpc}$, where MPC (Megapersec), is commonly used as unit of distance on the cosmic scale. $1 \mathrm{MPC}=3.26 \times 10^{6}$ light years. The message of the Hubble Law is that universe is expanding. This discovery led other scientists to conclude that the universe had a starting point which was singular in nature. The size of the universe is unknown and immeasurable, if it undergoes expansion or contraction, it is convenient to express distance at different epochs in terms of a cosmic scale $R(t)$ [1] The early universe was, it is thought, comprised of a soup of elementary particles with short mean free path. Ideally, to develop an understanding of the structure of the universe one would like to know the distribution of both the matter and light (radiation), to this point early universe has been described as uniform fluid of nearby constant velocity. Cosmology of recent times is based on two hypothesis and one law: 1) Universe is homogeneous 2) isotropic in large scale 3) Galaxies show a red shift in frequency approximately proportional to their distance. Coming back to the Vedic and Tanlik scriptural remark about manifestation and dissolution of the universe it is repeatedly said $[9,10]$ that at the time of dissolution of the universe it remain at a point like a gram seed and when the conditions are appropriate, manifestation starts again, subsequently after considerable time it is dissolved and the process go on like that periodically $[9,10]$. Our attempt in this paper is to examine the two parts i.e. profile of Cicer arietinum especially from the view point of spectrographic analysis and electron microscopic enlarged pictures of the two parts of a gram. Protein, amino acid contents etc as a whole for gram are available in some research papers [11] but separate profiles for two parts are not available yet. From spectrographic analysis made and others it is seen two parts are different at least in terms of arrangement. Lastly it is concluded what do they correspond to scientific analysis of Big Bang. Of course very recently many other non-singular theories like Teleparallel [12] theory, Loop Quantum cosmological theory i.e. inflationary \& deflationary, bounce scenarios in loop quantum cosmology, String cosmology \& Holographic principle, Balloon theory etc have been developed. But in this paper we shall restrict ourselves in Big Bang Singularity because Hubble Law of Red Shift was derived on the basis of observational spectroscopy of the galaxies and our analysis of two parts of gram a scriptural element.

\section{COSMOLOGICAL MODEL}

Main feature of the cosmological model is based on the assumption that cosmological objects, the vast particles sprang out from the point of Big Bang explosion are homogeneous and they obey the laws of the equation of state and laws of physics are assumed to hold good throughout the universe and the universe is 
homogeneous and isotropic in three dimensional space. One of the basic assumptions is the homogeneity and isotropy of the universe. The universe in large scale is observed to be homogeneous. The microwave back ground radiation, which is believed to be the relic of the early universe, is observed to be isotropic to a very high degree of accuracy on the large scales [5]. Assuming the universe as perfect fluid the cosmological equation is derived $[1,5]$. Main cosmological parameters and the equation $[1,5]$ are as under:

$$
\Omega_{0}+\Omega_{A}+\Omega_{k}=1
$$

where $\Omega_{0}=8 \pi G_{n} \rho_{0} 3 H_{0}^{2} . H_{0}=$ Hubble parameter and $G_{n}=$ Newton gravitational constant, $\rho_{0}$ is the mean energy density, $\Omega_{A}=A / 3 H_{0}^{2}, \Omega_{k}=-k / a_{0}^{2} H_{0}^{2}, \Omega_{k}$ in not an independent parameter, evolution of the universe there depends on the values of two other parameters, $\Omega_{0} \& \Omega_{\mathrm{A}}$. There is another parameter energy density of which evolves differently with time. Thus we can write [5]:

$$
\Omega_{0}=\Omega_{m}+\Omega_{r}
$$

$m \rightarrow$ mass and $r \rightarrow$ radiation. These two factors are very important. Actually, evolution of the universe is mainly governed by the two parameters $\Omega_{\mathrm{m}} \& \Omega_{\mathrm{r}}$ i.e. mass and radiation parameters. Also they bear an indication of whether it is an matter dominated or radiation dominated universe. Observational evidence indicate matter density $\gg$ radiation density i.e. $\Omega_{m} \gg \Omega_{r}$. Of course it is long after Big Bang explosion. Pressure $P$, mass density $\rho_{m}$ radiation density $\rho_{\mathrm{r}}$ plays an important role. And total density at a point is [6]:

$$
\rho=\rho_{m}+\rho_{r}
$$

One of the basic assumptions of standard Big Bang model of the universe is the homogeneity and isotropy of the universe. The universe in large scale is observed to be homogeneous. The microwave background radiation which is believed a relic of the early universe is observed [5] to be isotropic to a very high degree of accuracy on the large scale. In standard Big Bang Cosmology, neutrinos are the most abundant form of matter in the universe next to radiation. Regarding radiation, our visual perception of the universe comes from electromagnetic radiation and such radiation, at large are composed of varieties of frequencies which pervade the universe. In quantum view of light, it can be thought of as made up of individual particle-packet of energy-known as photon having zero mass and the total energy will be given by the kinetic energy i.e. $E=h f, h$ is plank constant. From the above discussion it is very clear that two important things the matter specially in the form infinitely small building block of subatomic quasi particles and the energy mainly in the form of radiation (photon) play important role in the evolution of the universe and remain dormant at a point of singularity before Big Bang explosion and when conditions are appropriate they start rolling ,manifestation starts and formation of heavenly bodies begin, In fact lost space and time in Big Bang singularity also resume its meaning. Of course it is differently explained in modified LandauRoychoudhury equations [13].

\section{SINGULARITY}

According to mathematical physics, singularity is a point in which a function takes infinite value, a singularity is in general a point at which a given mathematical object is not defined, or a point of an exceptional set where it fails to be well behaved in some particular way, as differentiability and so also is in geometry. We shall take of simplest case of mathematical and geometrical singularity.

For example, the function [14]

$$
f(x)=1 / x
$$

On the real line has a singularity at $x=0$, where it seems to explode to $\pm \infty$ and is not defined.

In our case

$$
f\left(\Omega_{0}\right)=1 /\left(\Omega_{m}+\Omega_{r}\right)
$$

Naturally, when $\Omega_{0}[1,5]$ takes the value of zero the function $f\left(\Omega_{0}\right)$ would become singular [14] i.e. 


$$
\left(\Omega_{m}+\Omega_{r}\right)=0
$$

i.e. when impact of matter and radiation become equal and opposite the function $f\left(\Omega_{0}\right)$ will be undefined $(\infty)$ and the universe will disappear and be specially in space-time when matter is infinitely dense (as the centre of black hole and in the point of Big Bang singularity).

There are other approaches [14] to the point of singularity particularly in real analysis left hand limit and right hand limit is of our interest where value $f\left(c^{-}\right)$is the value that the function $f(x)$ tends towards as the value $x$ approaches $c$ a specific value from below and the value $f\left(c^{+}\right)$is the value that the function $f(x)$ tends towards as the value $x$ approaches from above, regardless of the actual value the function has at the point where $x=c$. There are some functions for which these limit do not exist at all as approaches $c=0$ i.e. $\Omega_{0}$ tends towards zero. In fact they will be useful in describing the expanding and contracting universe with rest to the point of Big Bang singularity. Later we shall see what they mean in analysis of gram [15]. As black holes are playing very important role in manifestation and dissolution of the universe we will revisit the theory in short. One most intriguing prediction of Einstein's theory of General Relativity is the existence of Black Holes. A black hole [16] is a space time region where the gravitational field is so strong that no information carrying objects \& signals can escape it. A black hole is formed where the size of a gravitating object of mass $M$ becomes smaller than it, gravitational radius

$$
R_{s}=2 M G / C^{2}
$$

The boundary surface that restricts the non-return region is called the event horizon [16].

\section{ANALYSIS OF A SCRIPTURAL SAMPLE}

It is repeatedly mentioned in ancient Vedic and Tantric literatures $[9,10]$ that at the time of dissolution of the universe, it remain in the form of gram till the conditions are appropriate for manifestation again. On the basis of this scriptural remark an attempt has been made to study two parts of a gram specially by applying spectrographically available tools. In this connection it may be mentioned that lot of studies have already been made on Cicer arietinum for its contents particularly for the percentage of proteins, amino acid, carbohydrate and trace metals etc [11,15]. Sulphar protein in which we are interested for different order of liver in the universe [8] is also detected. Surprisingly any attempt has hardly been made to analyze two part of a gram separately for which we are interested how a tree remains concealed within two parts of a Cicer arietinum and to find a clue for Bing Bang Singularity as said in ancient scripture. An attempt has been made to analyze scientifically the two parts of a gram grain how they respond when different scientific tools are applied. Step by step results are as Figures 1-10.

Though the scriptural remark s have mentioned time and again that the universe remains as gram like form at the time of dissolution of the universe yet while discussing the matter with Sr. Professor cum scientists it was observed, they are of the opinion that idea will be applicable in other Cicer arietinum group seeds also and that is why other Cicer arietinum group elements like ground nut, France bean etc. which have two distinct parts in their seeds are included in picture at Figure 1. Further photograph at the Figure 2 of the gram as whole $\&$ in two parts was taken by the author using his own camera. Similarly electron microscopic pictures of two parts of a gram at Figure 3 to Figure 5 in different resolutions were taken by the author from the electron microscope of IACS to have better understanding of the two parts of a gram and the comprehensive understating idea of Big Bang Singularity of the universe as whole.

From the above analysis by applying different scientific techniques and Electron Microscopic pictures (vide Figure 1 to Figure 10 which are self explanatory) it is seen that the two parts of a same gram grain are not the same and but they are arranged differently. Though chemical analysis for the two parts of a gram grain has not been done, of course it may be extremely difficult due to smallness of the samples and difficulties of proper identification, yet electron microscopic pictures \& spectroscopic analyses give clues that they are different. Under a cover these two parts of gram with different characters are used to remain side by side with the potentiality of a big tree for a long time and when appropriate conditions are applied the tree is manifested. 


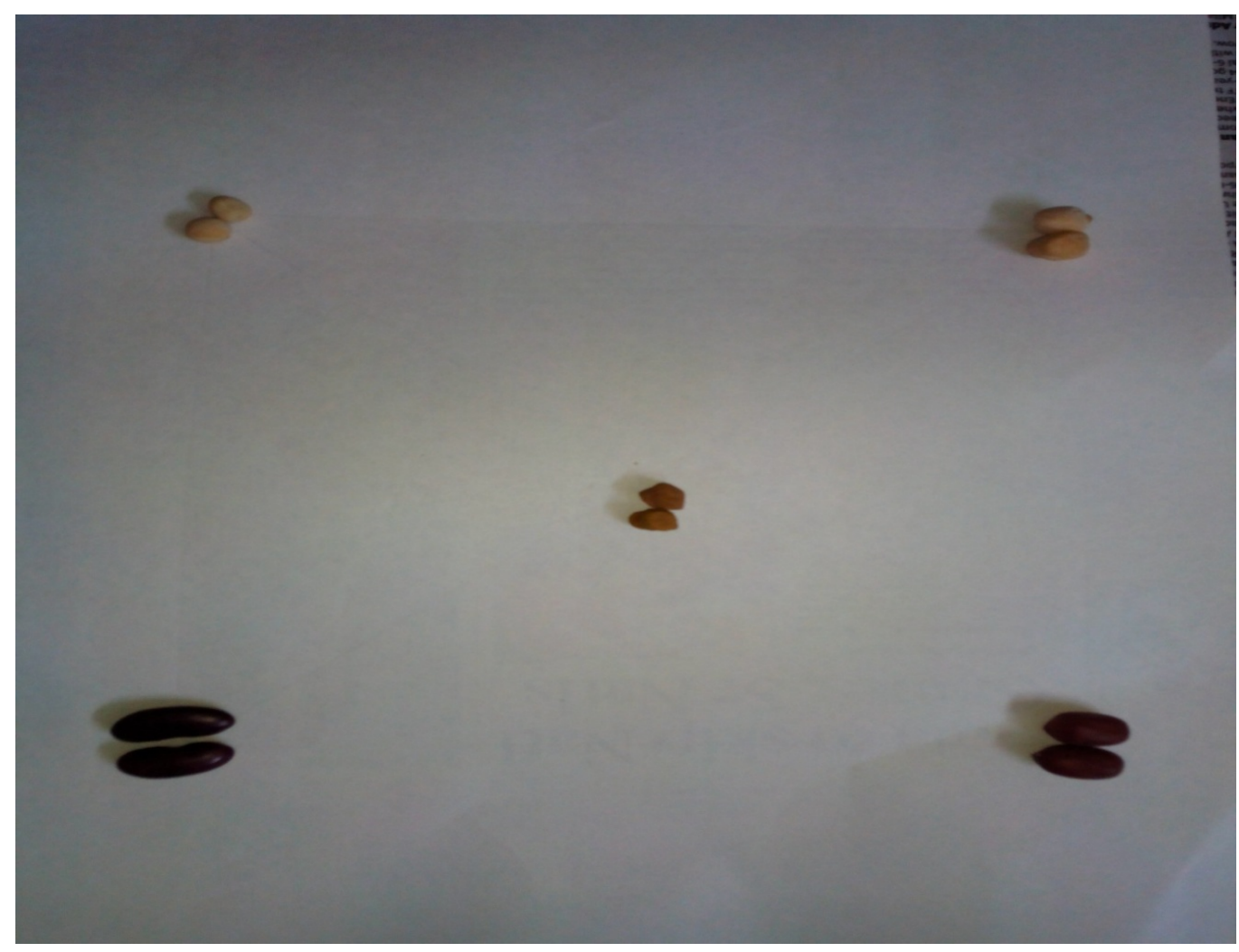

Figure 1. Normal photograph of different type of Cicer arietinum groups (i.e. gram, groundnut, red kidney bean etc.).

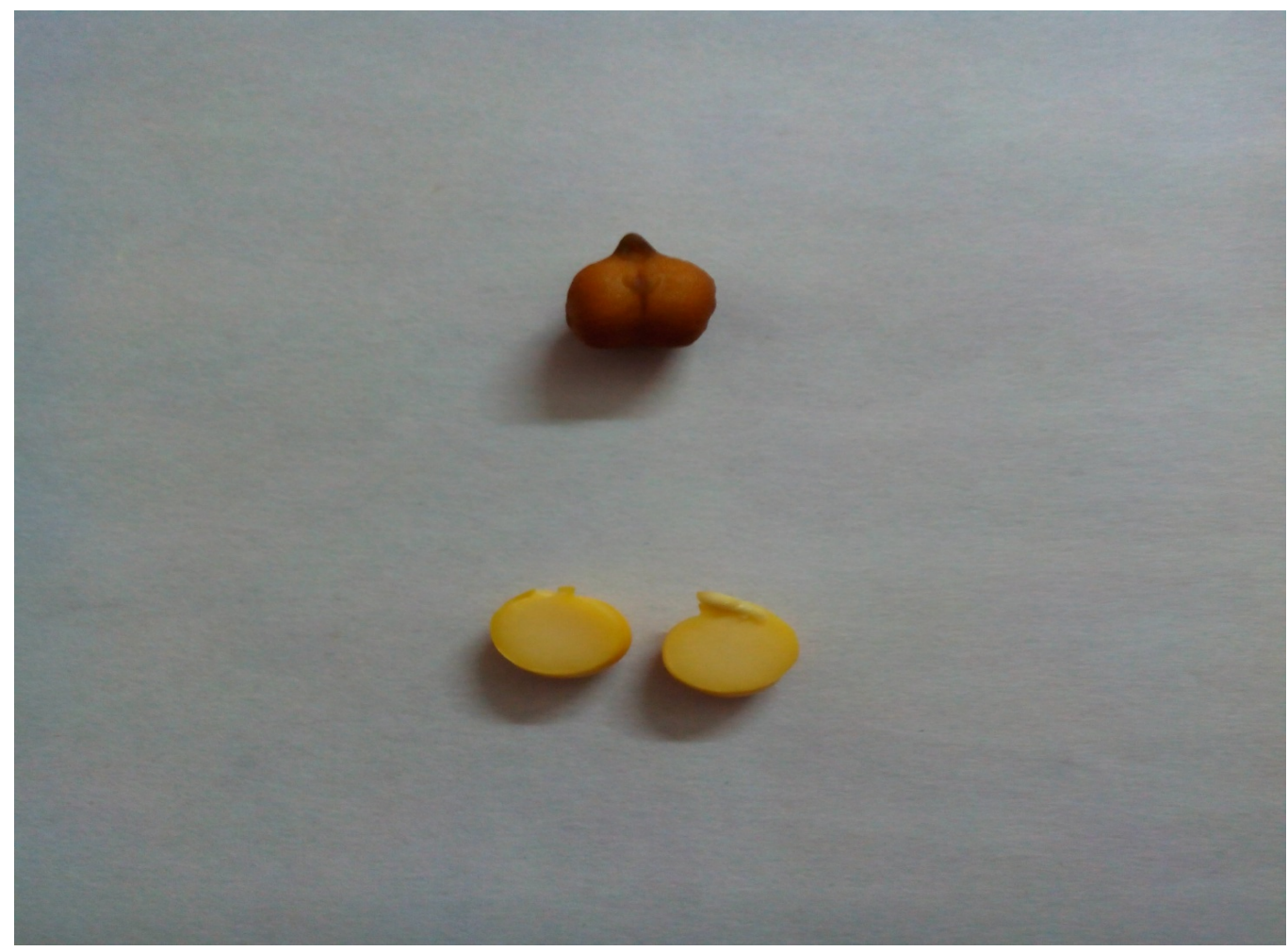

Figure 2. Normal photograph of one single gram as whole $\&$ in two parts. 


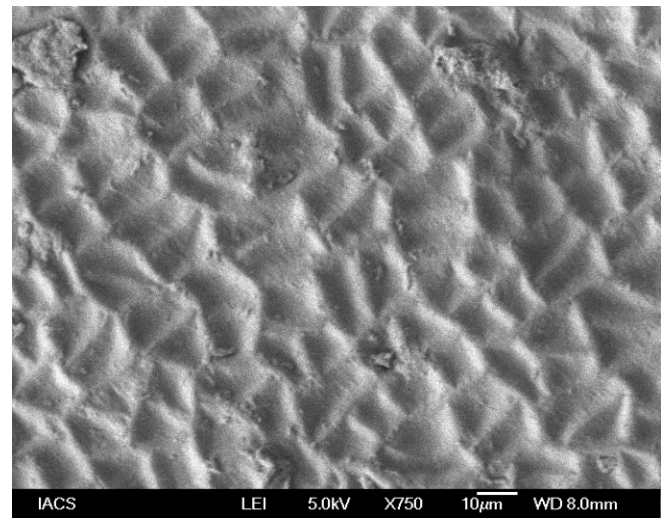

(a)

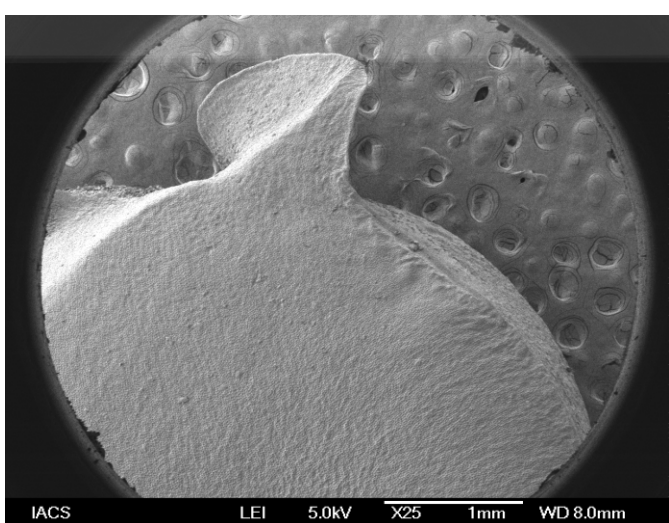

(b)

Figure 3. (a) \& (b) Electron microscopic picture right hand part of the gram grain in different resolution.

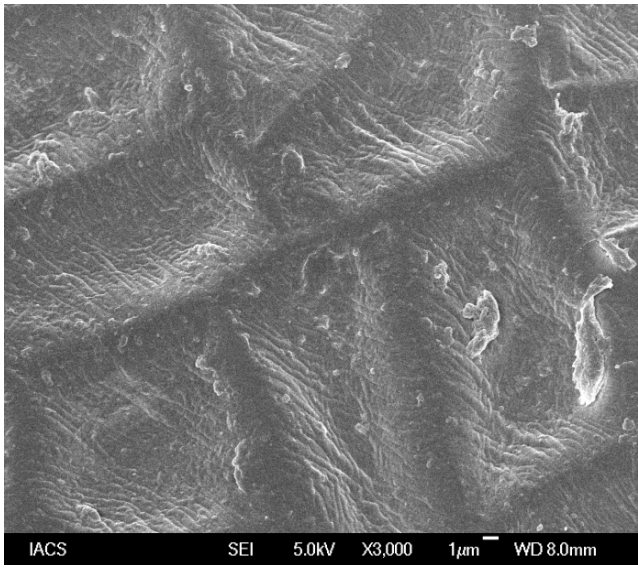

(a)

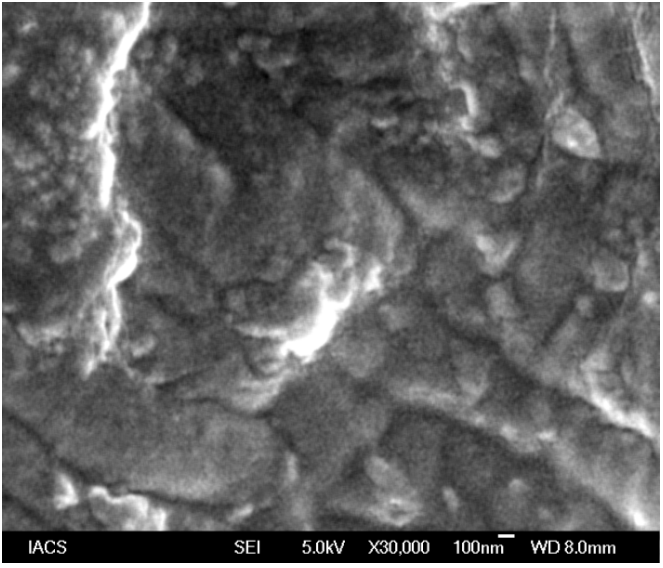

(b)

Figure 4. (a) \& (b) Electron Microscopic pictures of same part of a gram particle in different resolutions which is self-explanatory.

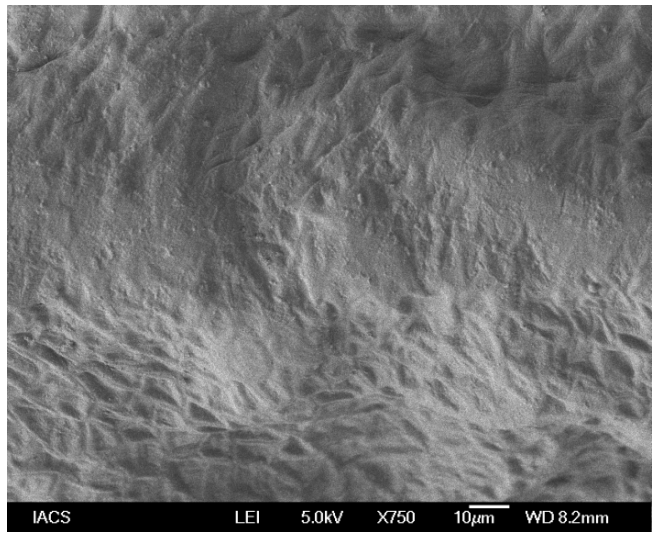

(a)

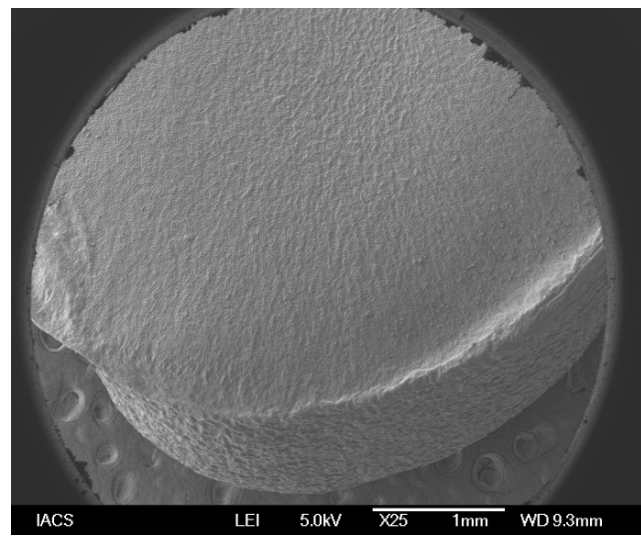

(b)

Figure 5. (a) \& (b) Electron Microscopic picture of the left hand side of the same gram particle in different resolutions. 


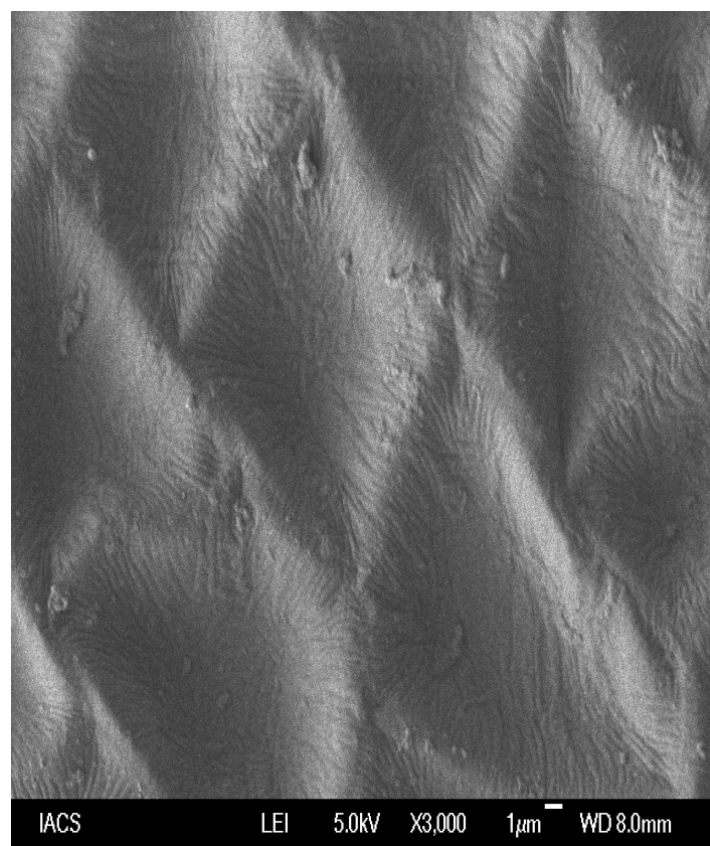

(a)

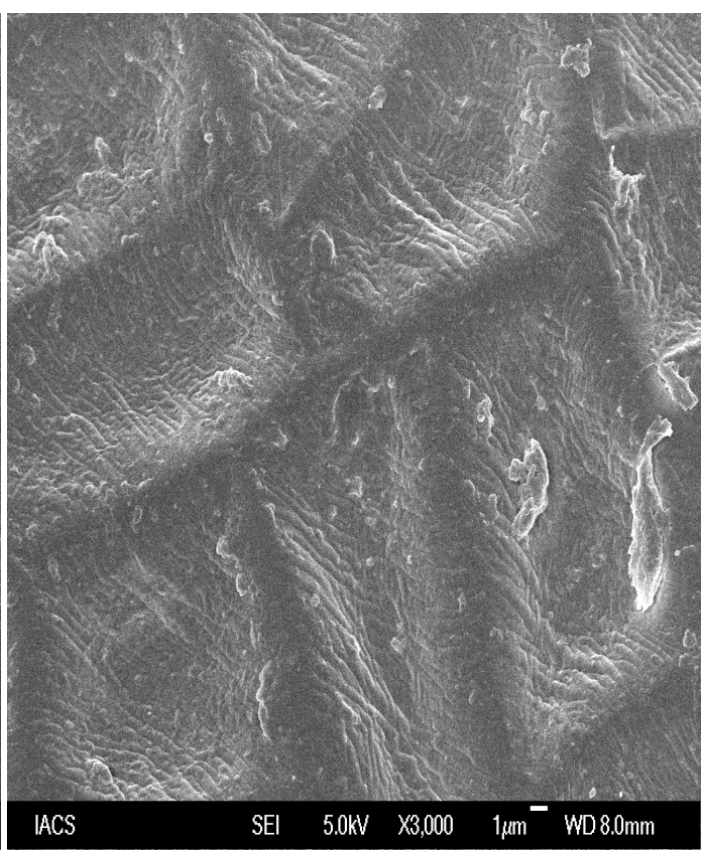

(b)

Figure 6. (a) \& (b) Electron Microscopic picture of left hand side \& right hand side of the gram at same resolution.

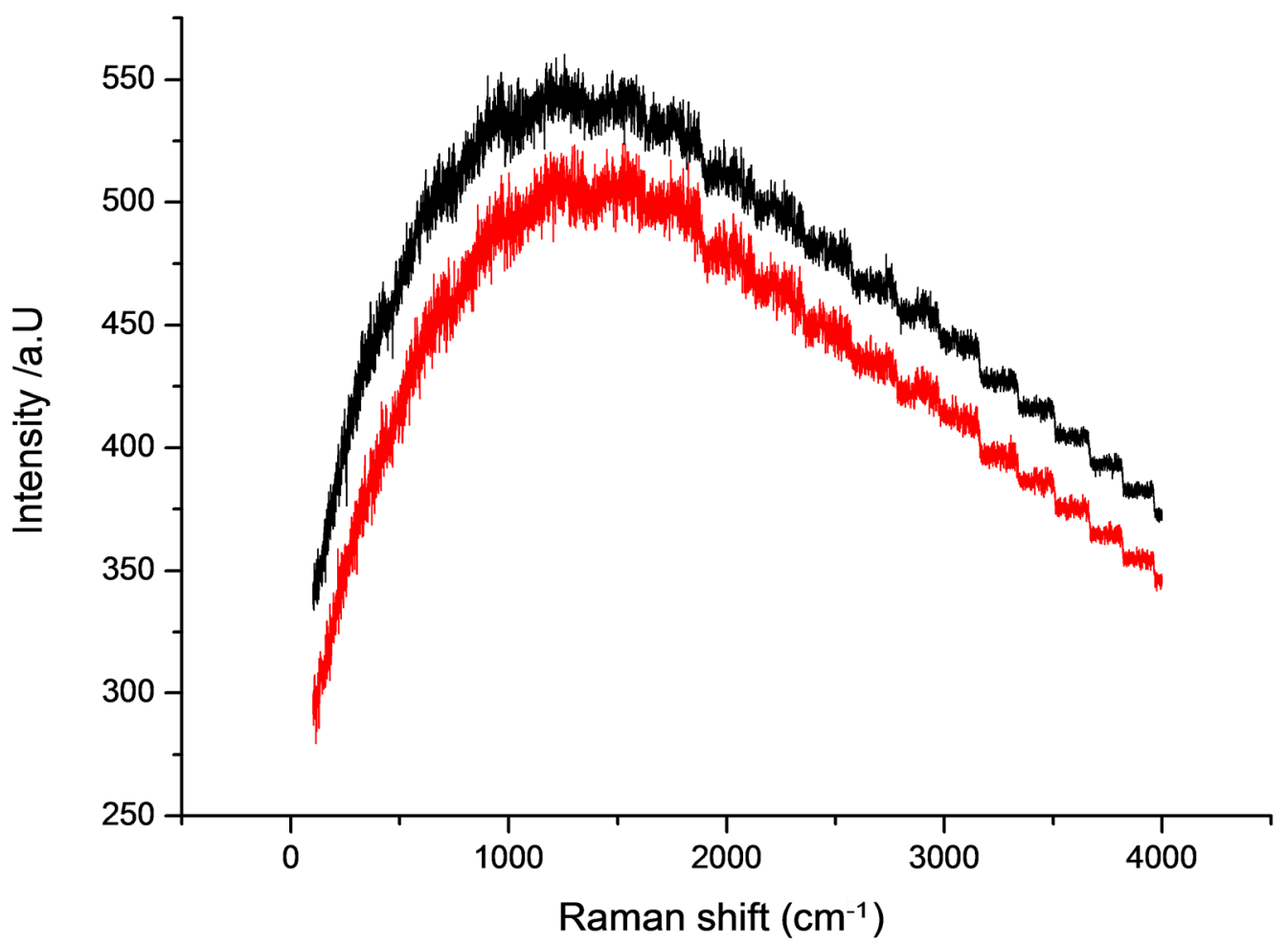

Figure 7. Graphical plotting Raman shift vrs intensity for two parts of a gram. (Left hand side and right hand side of gram show different intensity graphs with Raman shift. Observation was made at Raman Spectrometer of IACS). 


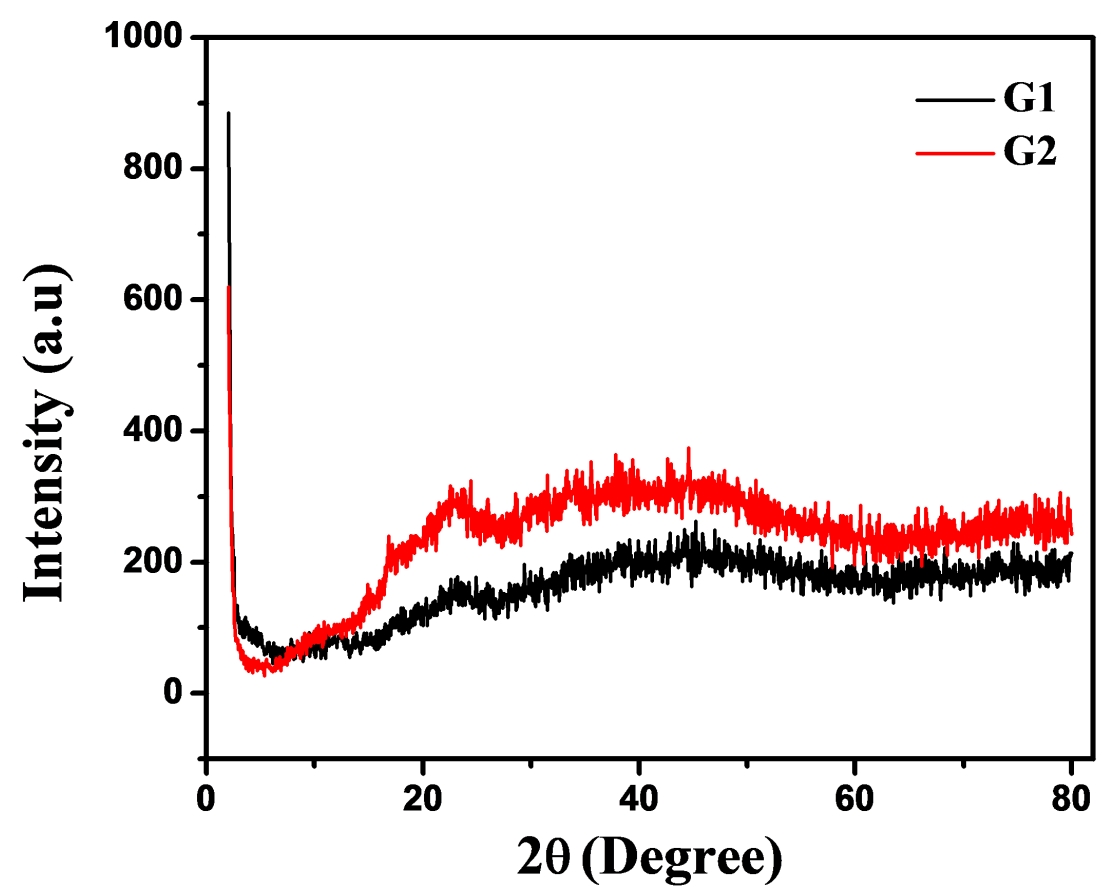

Figure 8. In X-ray powder diffraction method response of two parts of a gram.

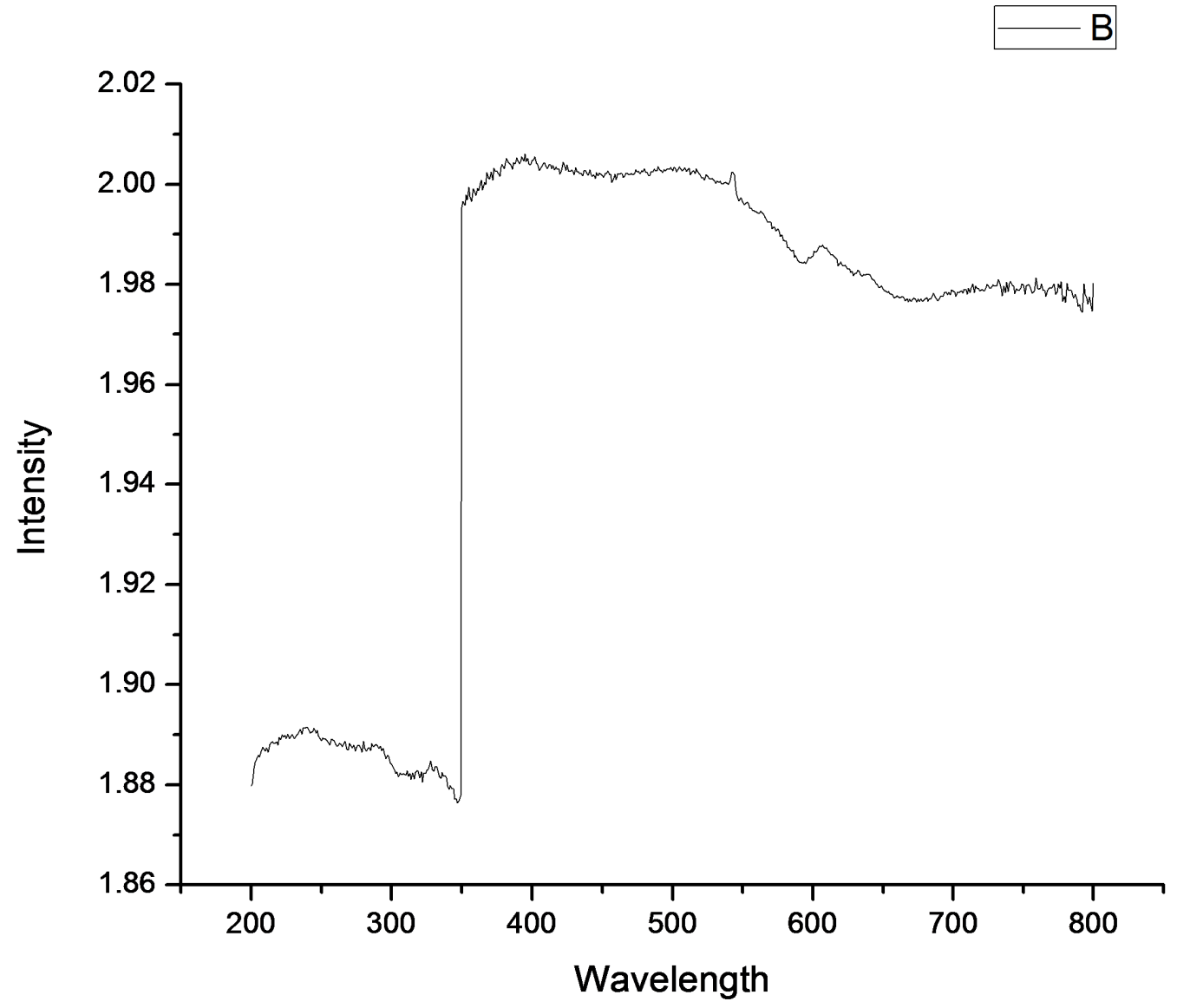

(a) 


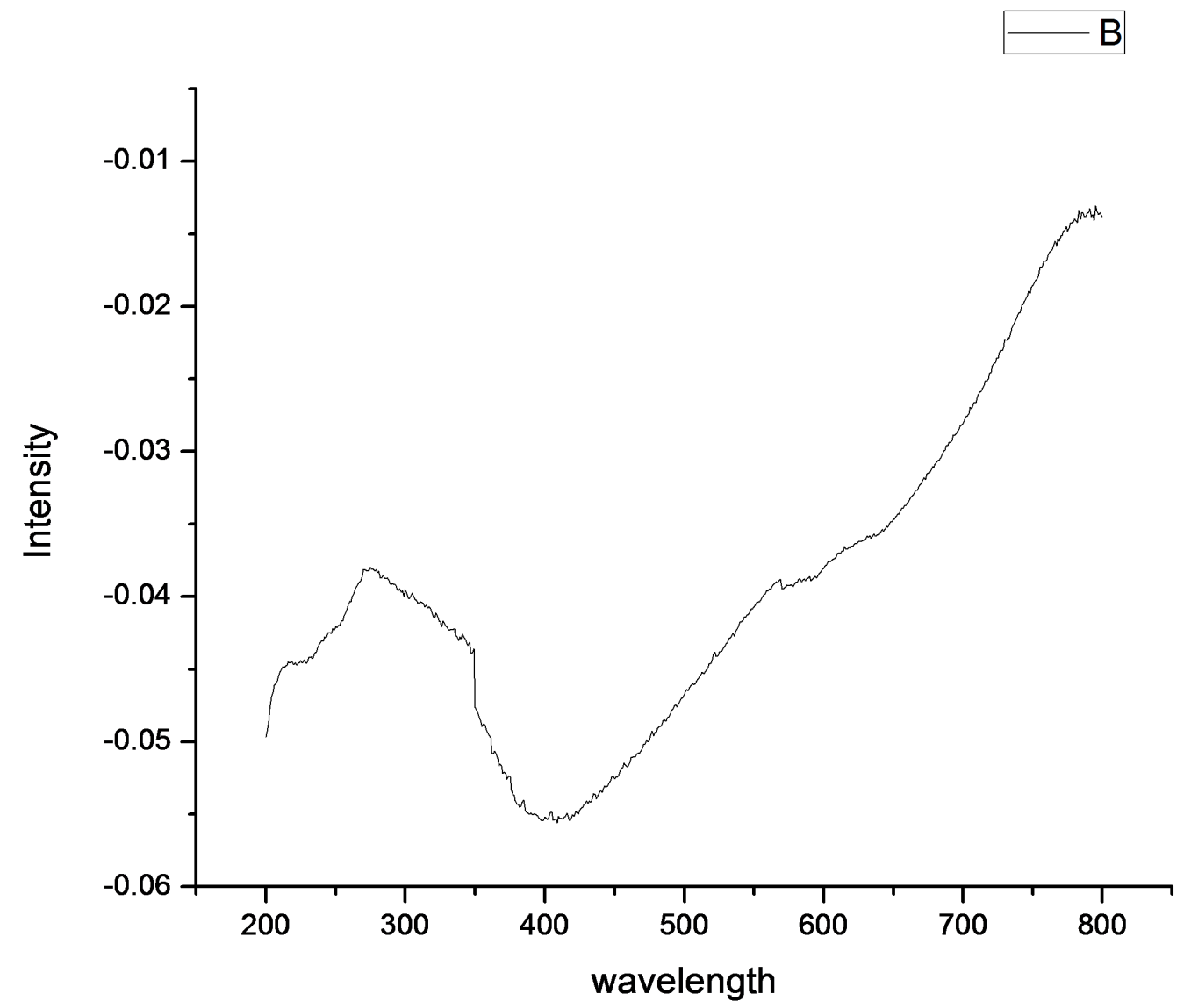

(b)

Figure 9. In UV (ultra violet) study the response of the two parts of the gram (wave length vrs intensity).

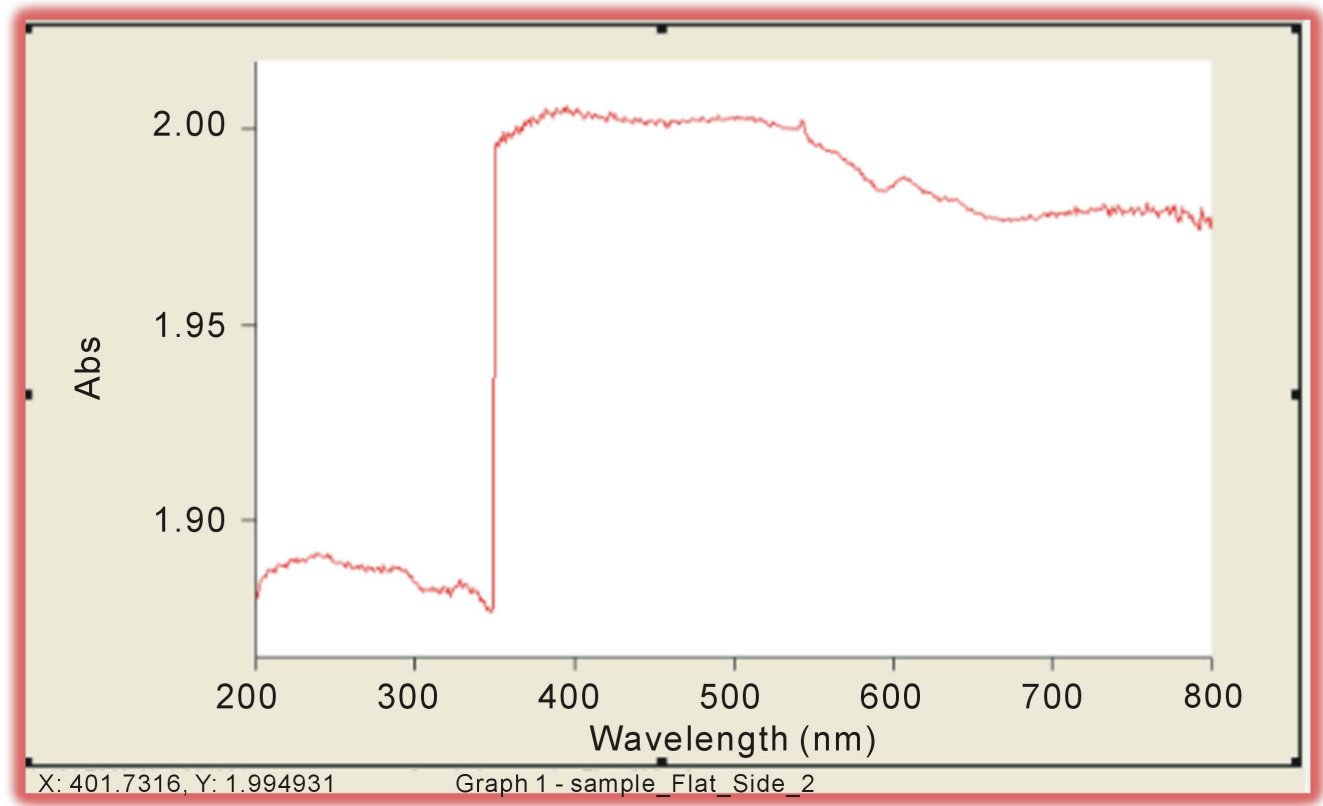

(a) 


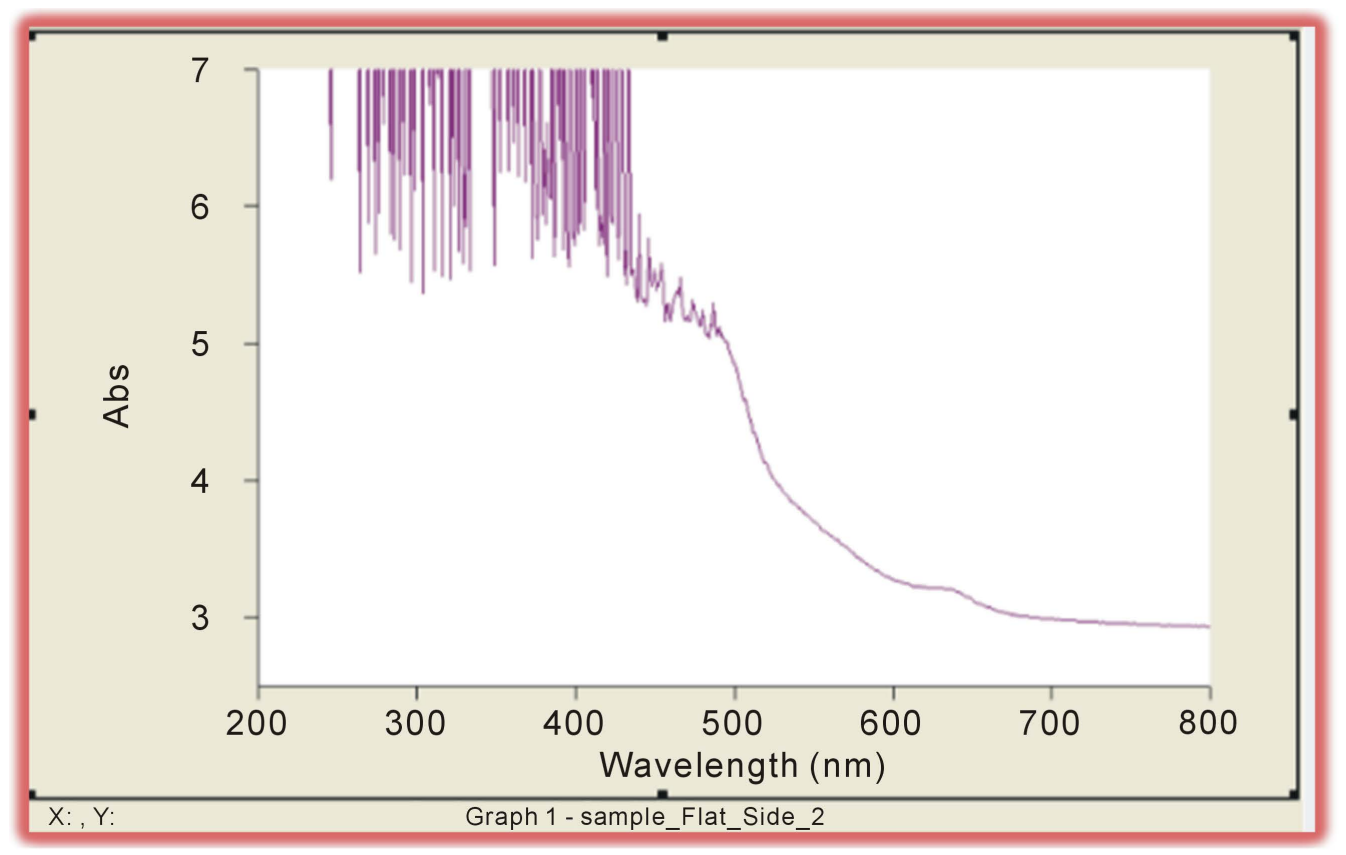

(b)

Figure 10. UV of the right hand side of the gram in different scale.

\section{DISCUSSION}

Entire universe remain concealed in a infinitesimally small Big Bang singular point in balance condition of infinitely dense matter and energy (wave form) [6] under the act of very strong Newtonian gravitational force that is in un-manifested state [1-6] and when this balance is disturbed at an ignition point tremendous energy mainly in the form of wave and the matter in the form of quasi-subatomic particles i.e. building block of the ultimate of sub-atomic particles [7] spring out at very high temperature. And with the cooling evolution of the universe starts step by step like a tree that come out from the two parts of the gram grain. At a certain point of evolution number of neutron stars and black holes exceed the certain limit when merger of two black holes by an effect as known Kick Effect [16] is started and so on by the reverse cascade process entire universe concentrates at a gram like singular point with two components of matter and radiation i.e. dissolution of the universe occurs under the act of the tremendous gravitational force. And from the electronic microscopic pictures at Figure 6(a) and Figure 6(b) at lower resolution of $1 \mu \mathrm{m}$ [17] it is seen that amino acid, protein etc happen to remain in loop form in two parts of a gram, of course, in different patterns. As such loop theory of the universe that the universe is woven in the form of loops, cannot be ruled out altogether. Again, when spectropic methods as available for analysis in solid state chemistry $[17,18-20]$ are applied on two parts of a gram it is found that spectra in X-ray powder diffraction method and Raman spectra plotted as function of intensity are similar in nature. The picture of Raman scattering of the two parts of a gram grain at picture of Figure 7 and the data (Photograph was taken from the Raman Spectrometer of IACS and the graphs for the two part of gram are self explanatory) it is seen that they behave slightly in different way side by side. Similar result i.e. graphs are obtained by applying X-rays powder diffraction method [17] at picture of Figure 8. Of course ultraviolet spectroscopy at pictures at Figure 9 of two parts of a gram shows different graphs at different range. Naturally responses of the two parts are somewhat similar for Raman spectra and X-ray powder diffraction method but the result is different for UV spectroscopy. In this connection it may not be out of the way to mention that the radiations, visible light, their luminosity, spectral shits of different light etc are the main source of information regarding heavenly bodies and the methods of analysis have been shown by different authors [17, 18-20]. This is the scenario for the scriptural sample. That means that the two parts of gram are not the 
same but behave differently. Similar will be the case for the Bing Bang point of singularity that the matter (building block of sub-atomic material particles) and the radiation (photons) would behave slightly in different way but remain in balance condition. In other words, building block of the sub-atomic material particles and high energy photons remains in infinitely small dense point side by side in balance condition i.e. in a point of singularity. One may be interested to know how these two parts of gram was prepared. In fact in night some good grams were kept in water about seven hours and in morning when they became soft, few grains were separated in two parts carefully and dried up in sunlight so that pairs did not mix up and preserved in small plastic rappers in pair. Here water is the internal condition and air is the external condition for coming up a tree from the gram seed. Again as microwave response on the two parts of a gram could not be taken up, Ultra Violet (UV) response at different scale was taken at the Fig 10 from the UV spectrometer of IACS

\section{CONCLUSIONS}

1) It is seen from the Electron Microscopic pictures of the two parts of Cicer arietinum and other spectroscopic responses that two part are not the same but they differ considerably but remain under an envelope side by side tightly until appropriate conditions are arose when tree comes out breaking the envelope of the scriptural element.

2) Similarly at the time of dissolution of the universe matter and radiation remain in their initial forms (i.e. ultimate building block of sub-atomic particles and ultimate intensive radiation with smallest possible wave length, so also charges carried by the quasi-subatomic particles remain in neutralized form by the action of opposite charges) exist in the point of Big Bang Singularity side by side under the act of tremendous gravitational force until appropriate condition arose when repulsive force generated counters the tremendous gravitational force, Big Bang explosion occur and the universe starts appearing again by the process of evolution. In this way the universe manifests again.

3) When impact of radiation and the matter parameters become equal and opposite the universe disappeared.

4) The idea that the universe is finally woven in the form of loops cannot be ruled out.

\section{ACKNOWLEDGEMENTS}

The author is grateful to the Indian Science congress Association for the award of Asutosh Mookerjee Fellowship (Sr Scientist). He is also acknowledge the help extended by teaching, non-teaching staffs \& some of the Research Scholars of the Indian Association for the cultivation of Science (IACS) to him for taking photograph, plotting of spectroscopic graphs etc in meaningful way. My special thanks go to Dr. Dilip Madak, Dr Sasanka Majhi, Dr. Manas Ghosh, Gour Basak, Shipra Ghosh,Sutapsh Dutta, Dr Babu Sona Sarkar and Dr Prashant Chandra Singh of IACS in this regard. My thanks also go to Prof Nanda Kumar Ghosh, Head and Prof. Arunav Chakraborty Department of physics Kalyani University (K.U.) for inspiration. My thanks also go to Prof. Sankar Narayan Sinha Head and Prof. Chandan Sengupta, Department of Botany K, U. for helpful discussion. Last of all I acknowledge the Co-operation extended by the Vice-Chancellor, K. U. by the way of giving me permission to continue research from the Department of Physics, K.U. Lastly my thanks go to the authority of Ramakrishna Mission Institute of Culture, Golpark, Kolkata and Jadavpur University for extending library facilities of rare books.

\section{REFERENCES}

1. Roos, M. (2015) Introduction to Cosmology. Wiley, West Sussex: Chennai.

2. Combes, F., et al. (2002) Galaxies and Cosmology. Springer, New York.

3. Srinivasan (2015) The Legacy of S Chandrasekhar (White Dwarf). The University of Chicago Press, Chicago, London.

4. Kalb, E.W. and Michel, S. (1990) Turner The Earlier Universe. West View, USA. 
5. Mahapatra, R.N. and Pal, P.B. (2014) Massive Neutrinos in Physics and Astrophysics: World Scientific Lecture Notes in Physics, Vol. 72. World Scientific Publishing Co Pte. Ltd., New Jersey, London, Singapore, Shanghai.

6. Liddle, A. (2003) An Introduction to the Modern Cosmology. John Wiley \& Sons Ltd., London.

7. Pagel, B.E.J. (2009) Nucleosynthesis and Chemical Evolution Galaxies. Cambridge University Press, Cambridge. https://doi.org/10.1017/CBO9780511812170

8. Sikdar, M.K. (2014) Different Order of Lives in the Universe. Indian Journal of Physics, 88, 767-776. https://doi.org/10.1007/s12648-014-0495-5

9. Smritithirtha, P.S.N. (1978) Nirvam Tantram. Nabha Bharat Publishers, Kolkata.

10. Prajnanda, S. (2010) Tantra Tatta Proveshika. Rama Krishna Vedanta Math, Kolkata.

11. Dhown, K., Malhotra, S., Dahiya, B.S. and Singh, D. (1991) Seed Proteins and Amino Acid Composition in Gram (Cicer arietinum). Plant Foods for Human Nutrition, 7, 225-232.

12. De-haro, J. and Amoros, J. (2013) Non Singular Models of Universe in Tele-Parallel Theories. Physical Review Letters, 110, Article ID: 071104. https://doi.org/10.1103/PhysRevLett.110.071104

13. Tawfik, A. and Diab, A. (2016) Emergence of Cosmic Space and Minimal Length in Quantum Gravity: A Large Class of Spacetimes, Equations of State, and Minimal Length Approaches. Indian Journal of Physics, 90, 1095-1103.

14. https://en.wikipedia.org/wiki/Singularity_(mathematics)

15. https://en.wikipedia.org/wiki/gram_floor

16. Frolov, V.P. and Zenikov, A. (2015) Introduction to Black Hole Physics. Oxford University Press, Oxford.

17. West, A.R. (2003) Solid State Chemistry and Its Application. John Wiley \& Sons Pte Ltd., Singapore.

18. Sikdar, M.K. (2017) On Rare Earths and Some Added Materials as By-Product. International Journal of Solid State Materials, $3,1$.

19. Sikdar, M.K. (1980) Racah Coefficients for Crystalline Solids. Progress of Theoretical Physics, 63, 72-83.

20. Rudra, P. and Sikdar, M.K. (1976) Tables of Clebsch-Gordan Coefficients of Magnetic Point Groups. Journal of Physics C: Solid State Physics, 9, 1. 